\title{
Transient Cavitation in Pre-Filled Syringes During Autoinjector Actuation
}

\author{
Jean-Christophe Veilleux*; Kazuki Maeda ; Tim Colonius ; Joseph E. Shepherd \\ California Institute of Technology, Pasadena, CA, USA
}

\begin{abstract}
Cavitation has been observed in the cone of a syringe actuated by an autoinjector device. Numerical simulations were used to determine if the cone can enhance the collapse of a bubble. We found the collapse of a bubble in a cone can be more violent than the collapse of a bubble close to a flat wall or in a free space due to the reflected wave focusing on the axis of symmetry.
\end{abstract}

Keywords: transient cavitation; tension waves; shock focusing; autoinjector; syringe

\section{Introduction}

Autoinjectors are used for emergency purposes and for long-term treatments requiring the frequent injection of biopharmaceuticals [1]. Even if the specific design of each autoinjector device may differ, in most devices currently available on the market, the mechanism is spring actuated [2,3]. Activation of the device may result in mechanical impacts between the moving components, and large accelerations/decelerations of the moving components may occur.

Figure 1 is a schematic of the internal components and actuation sequence of a SureClick ${ }^{\circledR}$ autoinjector. In panel $A$ the device is in its initial state just before actuation; the device bottom features are in contact with the patient's skin. In panel B the device has been activated; the spring-actuated driving rod is moving forward and impacts on the plunger-stopper. The impact of the driving rod on the plunger-stopper forces the plunger-stopper to start moving. This compresses and pressurizes the air gap (panel C) and sends a train of compression waves in the liquid from top to bottom. Because there is friction between the plunger-stopper and the syringe, the impact of the driving rod on the plunger-stopper also accelerates the syringe forward (panel C). The abrupt acceleration of the syringe creates a train of tension waves traveling from bottom to top in the syringe. In panel D, the syringe reaches its travel limit and is decelerated to a complete stop. The deceleration of the syringe creates a train of compression waves traveling from bottom to top in the syringe. In panel $\mathrm{D}$, the force exerted by the spring-actuated driving rod on the plunger-stopper pressurizes the syringe and the medicament is extruded through the needle.

Experimental measurements by Veilleux and Shepherd $[4,5,6]$ have shown the driving rod impacts on the plungerstopper at about $10 \mathrm{~m} / \mathrm{s}$, and the acceleration/deceleration of the syringe during device actuation can be as large as

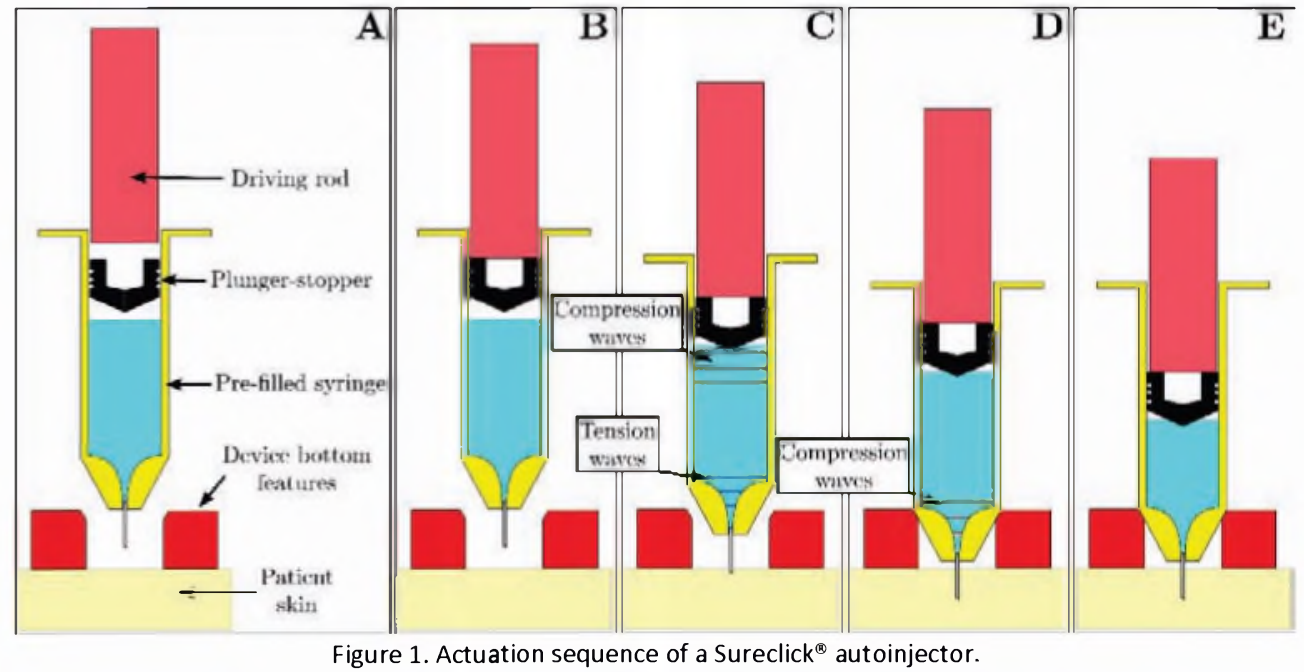

${ }^{1}$ SureClick $^{\circledR}$ is a registered trademark of Amgen.

*Corresponding Author, Jean-Christophe Veilleux: ic.veilleux@,caltech.edu 
$10,000-35,000 \mathrm{~m} / \mathrm{s}^{2}$. Such large accelerations of the syringe create tension waves with magnitudes large enough to reduce the liquid pressure to vapor pressure, and this results in cavitation in the syringe [4]. In particular, transient cavitation is consistently observed at the bottom of the syringe in the cone area.

Tension waves are also generated when compression waves reflect on the air gap located between the plunger-stopper and the liquid. Details on the complex interaction between the pressure waves. the air gap and the plunger-stopper are available in [7].

An example of the liquid pressure in the syringe during actuation is shown in figure 2. Events A through $E$ are clearly indicated. It is important to note that event $\mathrm{E}$ (extrusion of the drug) is only partially represented in figure 2; the extrusion continues beyond $6 \mathrm{~ms}$ for several seconds. The multiple pressure peaks shown in figure 2 between $0.8 \mathrm{~ms}$ and $2.0 \mathrm{~ms}$ are caused by the collapse of cavitation bubbles. In figure 3 it is possible to see the growth and collapse of a bubble within the cone upon acceleration of the syringe, and evidence of cavitation away from the cone. The time window over which frames 1 through 18 of figures 3 have been obtained is indicated in figure 2 (grey area - frame 1 corresponds to event A).

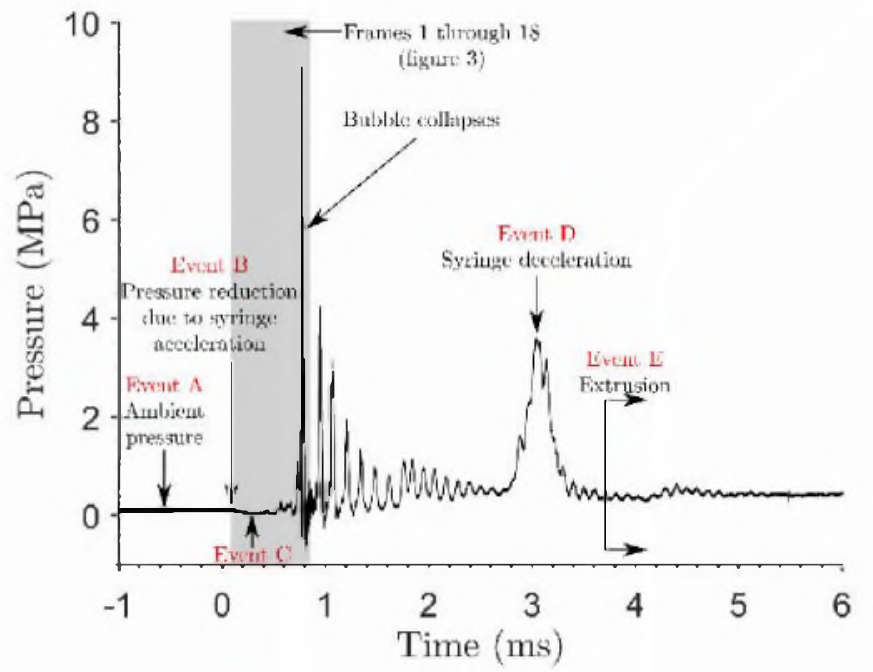

Figure 2. Liquid pressure during the transient events following actuation of a SureClick ${ }^{\circledR}$ autoinjector.
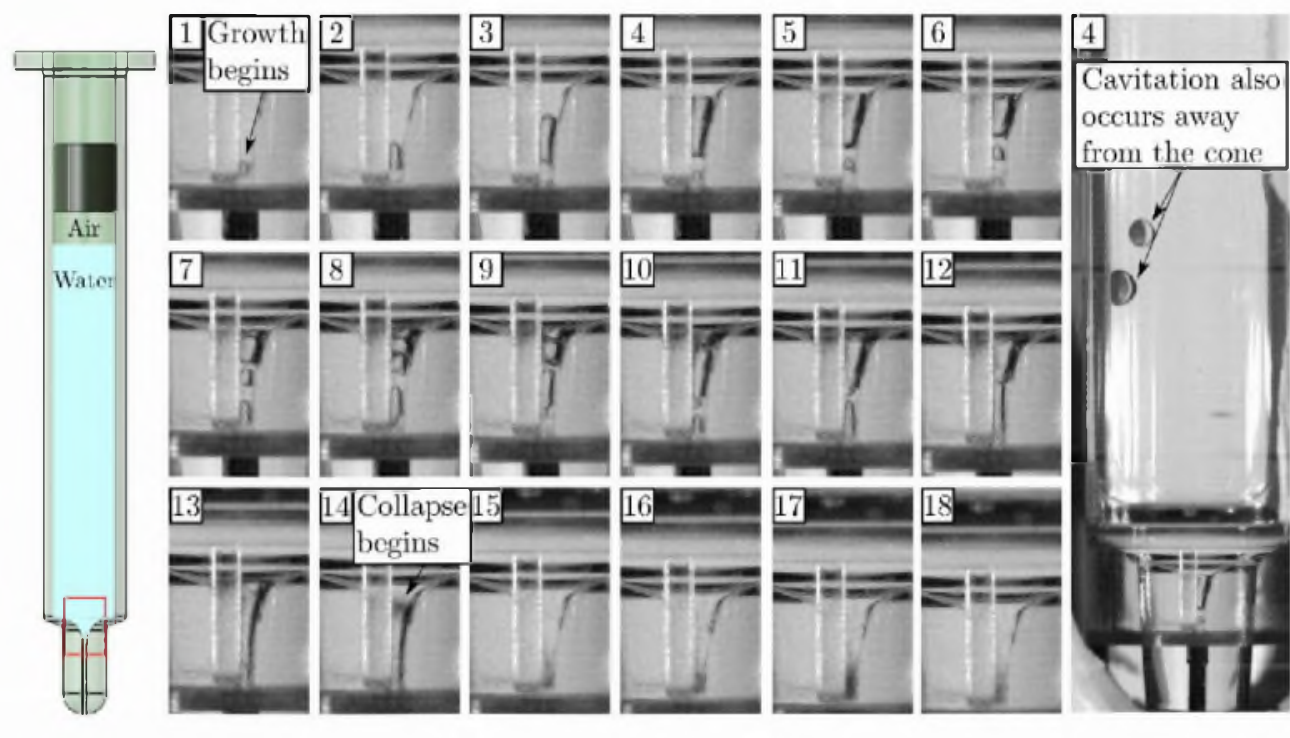

Figure 3. Sequence of images showing the growth and collapse of a bubble in the cone area of a syringe mounted inside a SureClick ${ }^{\circledast}$ autoinjector during acceleration of the syringe ( $30 \mu$ s between each frame). Frame 4 also shows evidence of cavitation away from the cone. 
Studying cavitation within the cone of a syringe mounted in an autoinjector is complicated by the small size of the syringe. Experiments on a scaled-up model autoinjector and numerical simulations have been used to gain insights. In this paper we report on the numerical simulations used to study the collapse of a cavitation bubble within the cone of the syringe. The main objective is to determine if and how the cone can enhance the collapse of a bubble.

\section{Numerical Setup}

The simulations have been performed with an interface-capturing method for compressible multi-component flows that employs an Eulerian finite-volume, weighted essentially non-oscillatory (WENO) scheme. The details about the numerical methods and verification/validation of the code are presented elsewhere $[8,9]$.

A first step in understanding the collapse of a bubble in the cone of a syringe is to study the shock-induced collapse of a gas bubble in an otherwise quiescent media, depicted in figure 4 . This configuration avoids having to address the randomness in nucleation events. Initially an air bubble of radius $R_{0}$ is located at a distance $H_{0}$ from the apex of a straight, water-filled cone of half-angle $\alpha$ and depth $L$. An incident weak shock is traveling down the cone. The pressure and density ahead of the shock are respectively $P_{0}$ and $\rho_{0}$. The pressure behind the shock is $P_{s}$ (other properties can be calculated with the shock-jump conditions). Inside the air bubble the initial pressure and density are respectively $P_{b, 0}$ and $\rho_{b, 0}$. At a later time, the weak shock has entered the cone (right-hand-side of figure 4); a reflected wave and a diffracted wave form. The pressure increases in the cone and the bubble is forced to collapse.

All numerical simulations reported in this paper were performed in 2D axisymmetric coordinates. A uniform grid is used in the shock-bubble interaction region, with a resolution of 108 cells per original bubble diameter. All quantities are normalized using $R_{0}, \rho_{0}$ and $a_{0}$. A stiffened gas equation of state is used for both fluids:

$$
P=(\gamma-1) \rho e-\gamma \pi_{\infty}
$$

where $P$ is the pressure, $e$ is the internal energy, $\rho$ is the density. The heat capacity ratio $\gamma$ and the normalized stiffness $\pi_{\infty}$ are respectively 1.4 and 0 for air, and 6.68 and 195.25 for water.

Shock-induced collapses have been simulated with $P_{s} / P_{0}=500, \rho_{\mathrm{b}, 0} / \rho_{0}=1.2 \mathrm{E}-3, L / R_{0}=10$, and $P_{b, 0}=P_{0}$. For comparison purposes, we also considered a Rayleigh collapse in free space where the bubble is initially set to a low pressure, $P_{b, 0}=0.002 P_{0}$ and the shock is not present $\left(P_{s}=P_{0}\right)$. Numerical simulations have been performed for $\alpha$ ranging from 25 to 60 degrees, and $H_{0} / R_{0}$ ranging from 1 to 10 .

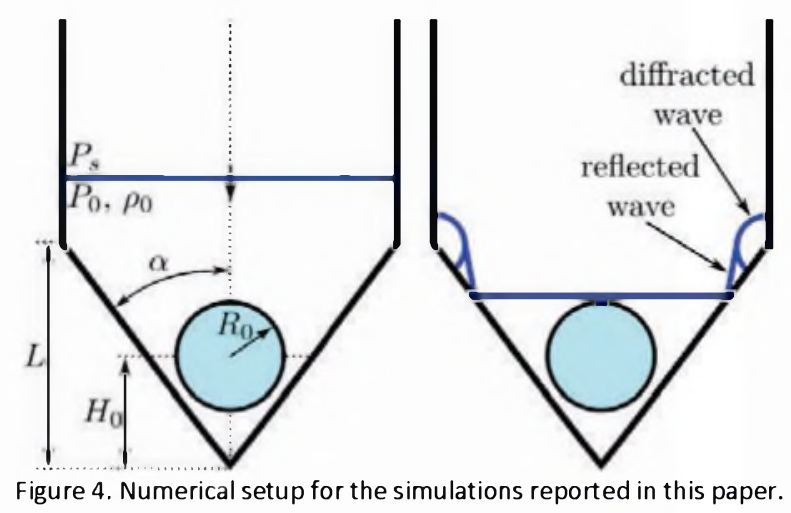

\section{Results \& Discussion}

The non-dimensional collapse time $\tau=a_{0} t_{\text {collapse }} / R_{0}$ is shown in figure 5 as a function of the offset distance for halfangles of $30,45,60$ and 90 degrees. The latter corresponds to the collapse of a bubble close to a flat wall. The collapse time for the free-space collapse (i.e., shock induced collapse of a bubble in a free space) and the Rayleigh collapse are also shown. As expected, the collapse time tends toward the free-space collapse time for large offset distances. This means the bubble does not feel the cone or the flat wall if the offset distance is large. When the offset distance is such that the bubble collapse is affected by the cone or the wall, the collapse time is generally reduced. This suggests the collapse is enhanced by the presence of the cone. The collapse time can even be less than the Rayleigh collapse. The 
minimum collapse time is achieved at an offset distance that decreases when the half-angle is decreased. Furthermore. the collapse of a bubble in a cone appears to be generally more violent than the collapse of a bubble close to a flat wall.

The peak pressure on the axis of symmetry is shown in figure 6 for the same cases shown in figure 5 . There are also 4 cases labeled as no bubble: these cases correspond to the situation where there is no bubble in the cone, and the peak pressure on the axis results from shock focusing alone.

The peak pressures on the axis appear to always be larger than for a free-space collapse, even when the bubble is far from the apex of the cone. The peak pressure on the axis is due to the combined effect of the collapsing bubble and shock focusing. Unless the offset distance is very small (i.e., $H_{0} R_{0} \sim 1$ ), the peak pressure on the axis is larger when the bubble is collapsing within a cone than when it collapses close to a flat wall. The collapse of a bubble within the cone gives large peak pressures for intermediate offset distances ranging between 3 and 6 . However, the peak axis pressure is not always larger than for the no bubble case. The same conclusions apply to the peak pressure on the wall (not shown in this paper) instead of the peak pressure on the axis.

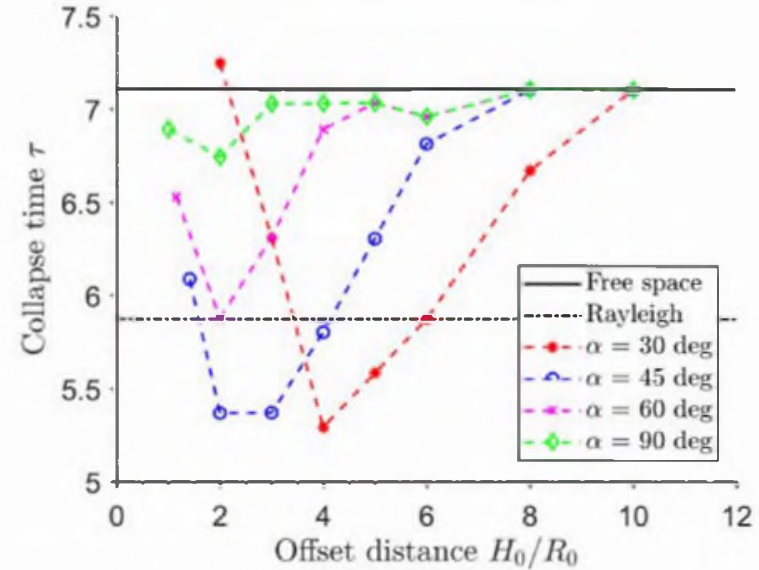

Figure 5. Collapse time of the bubble for various offset distances and half-angles.

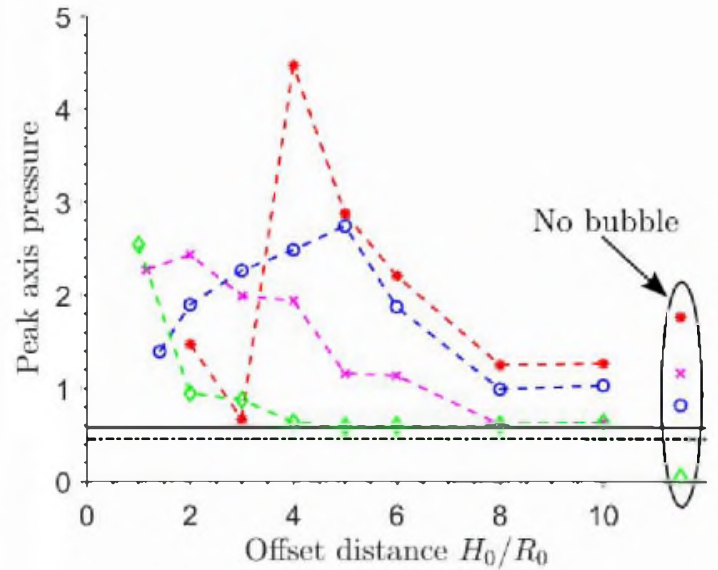

Figure 6. Peak pressure on the axis of symmetry for various offset distances and half-angles.

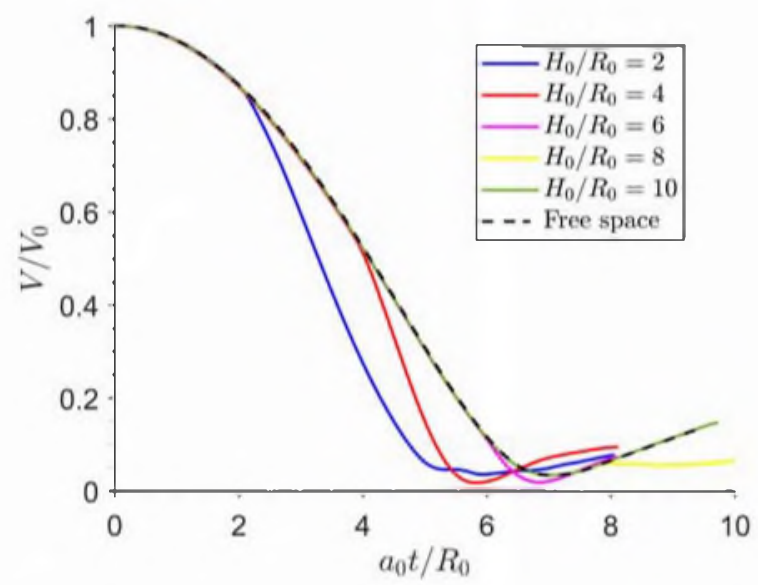

Figure 7. Bubble voulme history for various offset distances at a half-angle of 45 degrees.

The results above suggest the collapse of a bubble in a cone is generally more violent (i.e., smaller collapse time and larger peak pressures) than the collapse of a bubble close to a flat wall or a Rayleigh collapse. In order to gain insights about the mechanism of enhancement, we consider the bubble volume histories shown in figure 7 . The 
traces shown are for a half-angle of 45 degrees, and for a free-field collapse. Time 0 corresponds to the arrival of the incident wave on the proximal side of the bubble.

For all offset distances, the initial phase of the collapse is almost identical to the free-space collapse, before the cone influences the collapsing bubble. The effect of the cone begins at a time $a_{0} T / R_{0}$ which increases with the offset distance. This time $a_{0} T / R_{0}$ is well approximated by:

$$
\frac{a_{0} T}{R_{0}} \approx 2\left(\frac{H_{0}}{R_{0}}\right)(\sin \propto)^{2}
$$

For $a_{0} t / R_{0}>a_{0} T / R_{0}$ the volume history of a bubble collapsing in a cone differs greatly from that of the free-space collapse; the collapse is enhanced and happens more rapidly. The reflected wave is responsible for the enhancement of the collapse. In fact, if one assumes the reflected wave travels at sound speed and the bubble radius does not change between the arrival of the incident wave and the arrival of the reflected wave, it is possible to show that $2\left(H_{0} / R_{0}\right)(\sin \propto)^{2}$ corresponds exactly to the non-dimensional time it takes for the reflected wave to reach the bubble.

More details are provided in figure 8 for an offset distance of 4 and a half-angle of 45 degrees. The pressure field (top of each frame) and the numerical schlieren field (bottom of each frame) are shown. The bubble volume history is also shown along with the corresponding times for frames 1 through 7 (red, discontinuous lines).

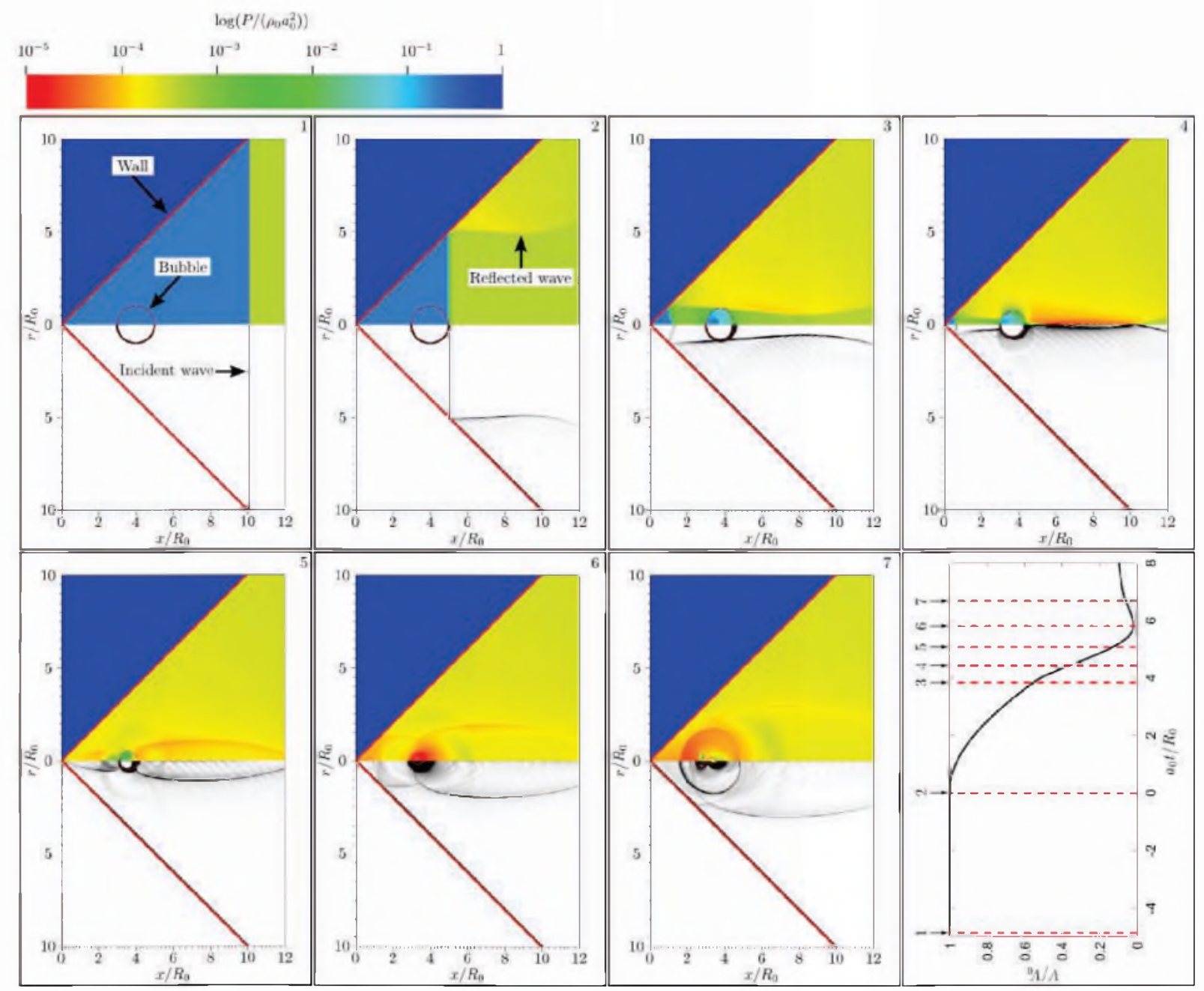

Figure 8. Sequence of images showing the collapse of a bubble in a cone for a half-angle of 45 degrees and an offset of 4. 
The incident and the reflected waves are indicated in frames 1 and 2 of figure 8 . Because the reflected wave converges toward the axis of symmetry the pressure rise it incurs is substantial. More interestingly, the enhancement of the collapse begins precisely when the reflected wave begins to interact with the bubble (frame 3 ). It turns out this is not a coincidence and the same observation can be made for all half-angles and offset distances.

If the non-dimensional collapse time $\tau$ is less than $a_{0} T / R_{0}-$ see. for example. offset distances of 8 and 10 in figure 7 - the reflected wave does not affect the collapsing bubble; the bubble collapses before the arrival of the reflected wave. This means the bubble does not feel the presence of the cone until after it has collapsed.

\section{Conclusion}

We experimentally observed the growth and collapse of cavitation bubbles within the cone of a syringe mounted inside an autoinjector device. Using numerical simulations, we were able to show the shock-induced collapse of a bubble in a cone can be more violent than the shock-induced collapse of a bubble located close to a flat wall or in a free-field. thus increasing the potential for wall damage.

The reflected wave which forms as the incident wave enters the cone is responsible for the enhancement of the collapse. The reflected wave converges on the axis of symmetry and creates a substantial pressure rise. The collapse is enhanced when the reflected wave begins to process the bubble. If the collapsing bubble is too far from the apex of the cone, the reflected wave will only reach the bubble after it has collapsed. Therefore, the collapsing bubble is affected by the conical section only if the non-dimensional collapse time $\tau>2\left(H_{0} / R_{0}\right)(\sin \propto)^{2}$.

\section{References}

[1] Akers, M.J. (2010). Sterile Drug Products: Formulation, Packaging, Manufacturing and Quality. Series: Drugs and the Pharmaceutical Sciences (Book 208). CRC Press.

[2] Thompson, I., Lange, J. (2013). Pen and Autoinjector Drug Delivery Devices. In Kohle, P. Shah, M. Rathore (Eds.) Sterile Product Development: Formulation, Process, Quality and Regulatory Considerations. Series: AAPS Advances in the Pharmaceutical Sciences Series (Volume 6). Springer-Verlag New York. DOI: 10.1007/978-1-4614-7978-9.

[3] French, D., Collins, J. (2010). Advances in Parenteral Injection Devices and Aids. In Nema, S. Ludwid, J. (Eds.) Pharmaceutical Dosage Forms: Parenteral Medications (Volume 3, pp. 71-85), $3^{\text {rd }}$ edition. CRC Press.

[4] Veilleux, J.-C., Shepherd, J.E. (2017). Dampers and Methods for Performing Measurements in an Autoinjector. U.S. Patent Application 15649329. Filed July 2017. Available online January 2018.

[5] Veilleux, J.-C., Shepherd, J.E. Understanding the Pressure and Stress Transients in Autoinjector Devices for Improved Designs and Reliability. Paper in preparation.

[6] Veilleux, J.C., Jazayeri, J., Eu, B., Shepherd, J.E. (2017). Primary Packaging \& Delivery Systems Interaction. In 10th Workshop on Monoclonal Antibodies of the Parenteral Drug Association, Berlin, Germany. DOI: 10.13140/RG.2.2.10549.37601.

[7] Veilleux, J.-C., Shepherd, J.E. (2017). Impulsively-Generated Pressure Transients and Strains in a Cylindrical Fluid-Filled Tube Terminated by a Converging Section. In Pressure Vessels and Piping Conference, Volume 4: Fluid-Structure Interaction. Waikoloa, Hawaii, USA. Paper PVP2017-65471. DOI: 10.1115/PVP2017-65471.

[8] Coralic, V., Colonius, T. (2014). Finite-volume WENO scheme for various compressible multicomponent flows. Journal of Computational Physics, 274, pp. 95-121. DOI: 10.1016/j.jcp.2014.06.003

[9] Meng. J.C. (2016). Numerical Simulations of Droplet Aerobreakup. PhD Thesis. Caltech. 